\title{
Multiplexed CRISPR/Cas9 genome editing increases the efficacy of homologous-dependent repair of donor sequences in mammalian cells
}

AUTHORS:

Ezio T. Fok ${ }^{1}$

Clement B. Penny ${ }^{1}$

Musa M. Mhlanga $a^{2,3}$

Marc S. Weinberg $4,5,6$

\section{AFFILIATIONS:}

${ }^{1}$ Medical Oncology Research

Unit, Department of Internal

Medicine, School of Clinical

Medicine, University of the

Witwatersrand, Johannesburg,

South Africa

${ }^{2}$ Gene Expression and

Biophysics Group, Synthetic

Biology-Emerging Research

Area, Council for Scientific and Industrial Research, Pretoria,

South Africa

${ }^{3}$ Unit of Biophysics and

Gene Expression, Institute of

Molecular Medicine, Faculty of

Medicine, University of Lisbon,

Lisbon, Portugal

${ }^{4}$ Antiviral Gene Therapy Research

Unit, Department of Molecular

Medicine and Haematology,

School of Pathology, University

of the Witwatersrand,

Johannesburg, South Africa

${ }^{5} \mathrm{HIV}$ Pathogenesis Research

Unit, Department of Molecular

Medicine and Haematology,

School of Pathology, University

of the Witwatersrand,

Johannesburg, South Africa

${ }^{6}$ Department of Molecular and

Experimental Medicine, The

Scripps Research Institute,

La Jolla, California, USA

\section{CORRESPONDENCE TO:}

Marc Weinberg

EMAIL:

marc.weinberg@wits.ac.za

\section{POSTAL ADDRESS:}

Antiviral Gene Therapy Research Unit, Department of Molecular

Medicine and Haematology,

School of Pathology, University of

the Witwatersrand Medical School,

7 York Road, Parktown 2193,

South Africa

\section{DATES:}

Received: 05 Jan. 2015

Revised: 04 Mar. 2015

Accepted: 23 Mar. 2015

\section{KEYWORDS:}

genome repair; genome targeting; sgRNA; HDR; T7 endonuclease I
Efficient and robust genome editing tools and strategies allow for specific and exact genetic changes to be captured in model systems, thereby accelerating both forward and reverse genetics studies. The development of CRISPR/Cas9 as a facile designer nuclease toolset has allowed for defined genetic modifications to be efficiently made through homology-directed repair of targeted DNA double-stranded breaks (DSBs) using exogenous repair templates. However, traditional single DSB strategies are still relatively inefficient as the short gene conversion tracts of mammalian cell systems limit the extent of achievable gene alteration from the DSB site. In order to improve on the inefficiency, we devised a dual cut strategy, which relies on reconstituting entire deleted gene fragments to precisely modify extensive gene regions of interest. Using the CRISPR/Cas9 system, we were able to introduce targeted deletions and repair of the endogenous KRAS gene locus in cell culture. The use of two simultaneous DSBs can be employed for efficient application of homology-directed repair with a large dsDNA donor sequence, thereby improving the efficacy of deriving cells with a desired gene editing outcome. In conclusion, a multiplexed CRISPR/Cas9 editing strategy represents an efficient tool for the editing of complex, heterologous sequence tracts.

\section{Introduction}

Precise genome editing represents a powerful new paradigm for both forward and reverse genetics studies in model systems. Sequence-specific nucleases have been used to expand our ability for precision engineering of the genome, and can be programmed to introduce targeted chromosomal double-stranded breaks (DSBs) to trigger endogenous DNA repair pathways. ${ }^{1-3}$ The error-prone non-homologous end joining (NHEJ) repair pathway involves the re-ligation of the broken DNA ends, and in the process introduces small mutagenic insertions and deletions (indels). The homology-dependent repair (HDR) pathway seamlessly repairs the damaged site by utilising a homologous template, a process which can be exploited for targeted gene modifications. ${ }^{4-6}$ These tools have enabled functional studies based on the systematic knockout and knockin of genes ${ }^{7}$, the modelling of human diseases in cell- or animal-based systems ${ }^{8-10}$, and the generation of isogenic cell lines for stable transgene expression ${ }^{11,12}$.

The clustered regularly interspaced short palindromic repeats (CRISPR) and the CRISPR-associated (Cas) protein system constitute an adaptive immune system found in prokaryotes, which functions to silence foreign DNA by RNA-guided nucleolytic digestion. ${ }^{13-15} \mathrm{CRISPR} / \mathrm{Cas}$ was adapted to target DNA by using short chimeric single-guide RNA (sgRNA) and a Cas9 nuclease reconstituted to function in cultured mammalian cells. ${ }^{16-19}$ The Cas 9 nuclease can be directed to a specific cognate DNA target via a 20-nucleotide 'guide' sequence within the sgRNA. The ease at which the nuclease can be reprogrammed by one or many sgRNAs has made this system highly effective for multiplexed genome editing applications in different cells $\mathrm{s}^{20-23}$ and eukaryotic model systems $\mathrm{s}^{22-35}$.

Conventional genome editing strategies make use of a single cleavage event to stimulate gene conversion with either ssDNA or dsDNA homologous repair templates. ${ }^{36}$ However, donor DNA sequences that are more distant from the DSB site, or which include heterologous sequences, are less efficiently repaired. ${ }^{37-39}$ This situation can be partially resolved by the simultaneous introduction of two separate DSBs at a targeted locus to induce deletion and replacement events over large genomic regions. ${ }^{26,27,40}$ However, the efficiency of this approach has not been determined and, to date, the HDR frequencies among approaches that apply single or multiplexed DSBS remain unknown. Using the CRISPR/Cas9 adopted from Streptococcus pyogenes, we exploited the capabilities of this system to induce DSBs to introduce an oncogenic KRAS (c.35G>T) mutant variant in a selectable human cell-based model system. We demonstrate that the facile introduction of two simultaneous DSBs improves the frequency, relative to a single DSB, of reconstituting dsDNA homologous donor sequences, but not ssDNA ODNs (oligodeoxynucleotides), at the desired locus. These results point to an improved approach for efficient HDR-mediated repair using the facile CRISPR/Cas9 editing system.

HOW TO CITE:

Fok ET, Penny CB, Mhlanga MM, Weinberg MS. Multiplexed CRISPR/Cas9 genome editing increases the efficacy of homologous-dependent repair of donor sequences in mammalian cells. S Afr J Sci. 2015;111(7/8), Art. \#2015-0002, 7 pages. http://dx.doi.org/10.17159/sajs.2015/20150002

(C) 2015. The Author(s).

Published under a Creative Commons Attribution Licence. 


\section{Materials and methods}

\section{Cell culture}

HEK 293 cells were maintained in Dulbecco's Modified Eagle Medium: F12 mixture containing glucose $(3.15 \mathrm{~g} / \mathrm{L})$, HEPES buffer $(15 \mathrm{mM})$ and L-glutamine (2.50 mM) (Lonza, USA), supplemented with $10 \%$ heat-inactivated foetal bovine serum (Hyclone, USA) and $0.2 \%$ penicillin-streptomycin antibiotic mix (10 $000 \mathrm{U} / \mathrm{mL})$ (Lonza, USA). The cells were grown in a $75-\mathrm{cm}^{3}$ flask and incubated in a $37^{\circ} \mathrm{C}$ humidified incubator with $5 \% \mathrm{CO}_{2}$.

\section{sgRNA assembly}

The sgRNA expression plasmid, pcDNA.H1.sgRNA, was generated by first using a H1.sgRNA gBlock sequence (Integrated DNA Technologies (IDT), Coralville, IA, USA) comprising two $B s m B I$ sites for facile guide sequence cloning using annealed oligos. The H1.sgRNA gBlock was Gibson Assembly cloned into the standard vector pcDNA3.1(+) (Life Technologies, CA, USA). For each of the five CRISPR/Cas9 target sequences, a pair of complementary oligonucleotides was chemically synthesised by IDT (USA):

sgRNA F1 224 forward: 5' - GATCCGAGTTTGTATTAAAAGGTAC - 3', sgRNA F1 224 reverse: 5' - AAACGTACCTTTTAATACAAACTCG - 3', sgRNA F2 225 forward: 5' - GATCCATGTGTGACATGTTCTAATA - 3', sgRNA F2 225 reverse: $5^{\prime}$ - AAACTATTAGAACATGTCACACATG - 3', sgRNA F3 226 forward: 5' - GATCCGTTTGTATTAAAAGGTACTGG - 3', sgRNA F3 226 reverse: 5' - AAACCCAGTACCTTTTAATACAAACG - 3', sgRNA R1 227 forward: 5' - GATCCGTGAATTAGCTGTATCGTCA - 3', sgRNA R1 227 reverse: 5' - AAACTGACGATACAGCTAATTCACG - 3', sgRNA R2 228 forward: 5' - GATCCACAAGATTTACCTCTATTGT - 3', sgRNA R2 228 reverse: 5' - AAACACAATAGAGGTAAATCTTGTG - 3'.

Each pair of oligonucleotides was composed of a 20-nucleotide guide sequence for their target and appropriate 5 ' overhangs that allowed for cohesive-end ligation into the $B s m B I$ (Thermo Scientific, USA) digested pcDNA.H1.sgRNA expression plasmid. Sanger sequencing was performed to validate the insertion of the guide sequence into the sgRNA expression plasmid.

\section{T7 Endonuclease I assay}

At $24 \mathrm{~h}$ prior to transfection, HEK 293 cells were seeded at a density of $250000 \mathrm{cells} / \mathrm{well}$ in a six-well plate. The cells were transfected with $2 \mu \mathrm{g}$ of the sgRNA expression plasmid and $2 \mu \mathrm{g}$ of the Cas9 expression plasmid (pX330) (Addgene plasmid \#42230) ${ }^{16}$ using $12 \mu \mathrm{L}$ of branched polyethylenimine (Sigma Aldrich, USA). At $48 \mathrm{~h}$ posttransfection, the cells were harvested and the gDNA was extracted from these cells using the QIAamp DNA Mini Kit (Qiagen, Germany). Genomic regions surrounding the CRISPR/Cas9 target sites were amplified from this gDNA with primers for the $5^{\prime}$ cleavage region $\left(5^{\prime} K R A S\right.$ forward: $5^{\prime}$ - CGCAGAACAGCAGTCTGGC - $3^{\prime}$ and 5' KRAS reverse: 5' CTACGCCACCAGCTCCAAC - $3^{\prime}$ ), and the $3^{\prime}$ cleavage region ( $3^{\prime}$ KRAS forward: 5' - AAGGCCTGCTGAAAATGACTG - $3^{\prime}$ and $3^{\prime}$ KRAS reverse: 5' - GCACAGAGAGTGAACATCATGG - 3'). The T7 Endonuclease I (T7E1) assay was performed as previously described. ${ }^{36,41}$ Briefly, $600 \mathrm{ng}$ of DNA amplified from the genomic target region was denatured and annealed to form heteroduplexes. Amplicons $(150 \mathrm{ng})$ were digested with $3 \mathrm{U}$ of T7E1 (New England Biolabs, Germany) and resolved on an $8 \%$ polyacrylamide gel stained with SYBR Green I Nucleic Acid Gel Stain (Invitrogen, USA).

\section{Micro-deletion detection}

HEK 293 cells were transfected using polyethylenimine with $2 \mu \mathrm{g}$ of the $5^{\prime}$ cleavage sgRNA expression plasmid, $2 \mu \mathrm{g}$ of the $3^{\prime}$ sgRNA expression plasmid and $2 \mu \mathrm{g}$ of $\mathrm{pX} 330$. gDNA was extracted using the QIAamp DNA Mini Kit (Qiagen, Germany) 48 h post-transfection. Polymerase chain reaction (PCR) amplification of the deletion junction was performed using Phusion Flash High-Fidelity PCR Mix (Thermo Scientific, USA) with an initial denaturation at $98{ }^{\circ} \mathrm{C}$ for $10 \mathrm{~s}$, followed by 35 cycles of denaturation at $98^{\circ} \mathrm{C}$ for $1 \mathrm{~s}$, annealing at $65.4^{\circ} \mathrm{C}$ for $5 \mathrm{~s}$, extension at $72^{\circ} \mathrm{C}$ for $10 \mathrm{~s}$ and a final extension at $72^{\circ} \mathrm{C}$ for 1 min using the $5^{\prime}$ KRAS forward and $3^{\prime} K R A S$ reverse primers described in the T7E1 assay. The PCR products were then visualised on an ethidium bromide $(0.5 \mu \mathrm{g} / \mathrm{mL})$ stained TAE agarose gel $(2 \%(\mathrm{w} / \mathrm{v}))$.

\section{Restriction fragment length polymorphism analysis for detection of HDR}

HEK 293 cells were transfected with $2 \mu \mathrm{g}$ of sgRNA expression plasmid, $2 \mu \mathrm{g}$ of $\mathrm{pX} 330$ and $2 \mu \mathrm{L}$ of the ssODN repair template $(100 \mu \mathrm{M})$ for the single cut strategy. For the dual cut strategy, $2 \mu \mathrm{g}$ of each sgRNA expression plasmid, $2 \mu \mathrm{g}$ of $\mathrm{pX} 330$ and $2 \mu \mathrm{L}$ of the ssODN repair template $(100 \mu \mathrm{M})$ were used. After $72 \mathrm{~h}$, the gDNA was extracted from these transfected cells and the repaired region was amplified using PCR with the $5^{\prime}$ KRAS forward and $3^{\prime}$ KRAS reverse primers (described for the T7E1 assay). The PCR products were purified and $300 \mathrm{ng}$ of the DNA was digested with EcoRI (Thermo Scientific, USA). The products were resolved and visualised on a $8 \%$ polyacrylamide gel which was stained with SYBR Green I Nucleic Acid Gel Stain (Invitrogen, USA).

\section{dsDNA donor sequences}

The SSDNA ODN sequences used were chemically synthesised (IDT, USA) (EcoRI site underlined):

F1 SSODN: $5^{\prime}-$

TTGAAATAATTTTTCATATAAAGGTGAGTTTGTATTAAAAGGGAATTCTACTGG TGGAGTATTTGATAGTGTATTAACCTTATGTGTGAC

R2 sSODN: 5'-CAGCTAATTCAGAATCATTTTGTGGACGAATATGATCCAACA GAATTCATAGAGGTAAATCTTGTTTTAATATGCATATTACTGGTGCAG F1+R2 ssODN: 5'-TTGAAATAATTTTTCATATAAAGGTGAGTTTGTATTAA\AA GGGAATTCATAGAGGTAAATCTTGTTTTAATATGCATATTACTGGTGCAG.

The dsDNA donor construct was assembled by fusing five PCR products in an overlap extension PCR:

eGFP F: $5^{\prime}$ -

TCAAGTTGGCGGGAGACGTCGAGTCCAACCCTGGGCCCATGGTGAGCAAG GGCGAGGAGC - 3'; eGFP R: 5' -

CACACAAAAAACCAACACACAGATGTAATGAAAATAAAGATATTTTATTTCTAG AGTATACGGACCGTTACTTG - 3'; 5' HA F 5' -

GTTTGTATTAAAAGGTACTGGTGGAGTATTTGATAGTGTATTAACCTTATCACA

CAAAAAACCAACACACAGATG - 3'; NeoR F 5' -

TAATGAAAATGTGACTATATTAGAACATGTCACACGCGC

AGCACCATGGCCT GAAATAAC - 3'; NeoR 5' -

ACGTCTCCCGCCAAC

TTGAGAAGGTCAAAATTCAAATCTGTTTCACGAAG AACTCGTCAAGAAGG CGATAGAAG - 3'; KRAS F: 5' -

GTGTGACATGTTCTAATATAGTCACATTTTCATTATTTTTATTATAAGGCCTGCT GAAAATGACTGAATATAAACTTGTGGTAGTTGGAG - 3'; KRAS R1: 5' GTCTACAAAATGATTCTGAATTAGCTGTATCGTCAAGGCACTCTTGCCTACGC CAACAGCTCCAACTACCA CAAGTTT - 3'; KRAS R2: 5' -

GCATATTAAACAAGATTTACCTCTATTG TTGGATCATATTCGTCTACAAAATG ATTCTG - 3'; 5' HA F2: 5' -

TAGCTGTTGCATATTGACTTCTAACACTTAGAGG - 3'; 5' HA R2: $5^{\prime}$ ATAAGGTTAATACACTATCAAATACTCCACCAGTACC - 3'; 3' HA F2: 5' AATCATTTTGTGGACGAATATGATCCCACAAT - 3'; 3' HA R2: $5^{\prime}$ CCATCAAACAATTATATTTCACTAGTACAATTAAATCTAACCTTT - 3 '.

The plasmid pCl-NeoR-EGFP was used as a template for SV40 driven NeoR. Amplicons comprising the $5^{\prime}$ and $3^{\prime}$ homology arms, KRAS sequence and SV40-Neo-2A-eGFP sequences were assembled by overlap-extension PCR. The dsDNA donor was ligated into the pJet1.2/blunt Cloning Vector from the CloneJet PCR Cloning Kit (Thermo Scientific, USA) and subsequently sequenced. The dsDNA donor was linearised by PCR amplification using Phusion Flash High-Fidelity PCR Mix (Thermo Scientific, USA) and the 5' HA F2 and 3' HA R2 primers, with an initial denaturation step at $98^{\circ} \mathrm{C}$ for $10 \mathrm{~s}$, followed by 35 cycles of denaturation at $98^{\circ} \mathrm{C}$ for $1 \mathrm{~s}$, annealing at $62.7^{\circ} \mathrm{C}$ for $5 \mathrm{~s}$, extension at $72{ }^{\circ} \mathrm{C}$ for $45 \mathrm{~s}$ and a final extension at $72^{\circ} \mathrm{C}$ for $1 \mathrm{~min}$. This linear dsDNA 
donor construct was column purified with the GeneJet PCR Purification Kit (Thermo Scientific, Lithuania).

\section{Quantitative real-time PCR}

HEK 293 cells were transfected with $2 \mu \mathrm{g}$ of each sgRNA plasmid, $2 \mu \mathrm{g}$ of pX330 and $1 \mu \mathrm{g}$ of the linear dsDNA donor PCR product. Half of the transfected cells in each well were harvested for gDNA extraction $72 \mathrm{~h}$ post-transfection, and the remaining cells were maintained for another 14 days. Quantitative real-time PCR (qPCR) was performed using SYBR Green PCR Mastermix (Applied Biosystems, USA) on the Applied Biosystems 7500 RT PCR System, which was programmed for an initial hold stage at $50{ }^{\circ} \mathrm{C}$ for $2 \mathrm{~min}$, followed by denaturation at $95^{\circ} \mathrm{C}$ for $10 \mathrm{~min}$ and 50 cycles of denaturation at $95^{\circ} \mathrm{C}$ for $15 \mathrm{~s}$ and annealing and extending either at $55^{\circ} \mathrm{C}$ (for primer set $\mathrm{A}, \mathrm{B}$ and $\mathrm{B}$-actin) or at $60^{\circ} \mathrm{C}$ (for primer set $\mathrm{C}$ ) for $1 \mathrm{~min}$. An inter-plate calibrator reaction was included for each primer set to control for any technical variations between the 72-h and 14-day samples. The inter-plate calibrator template mixture was made up by adding $10 \mu \mathrm{L}$ of each gDNA sample $(16 \mathrm{ng} / \mu \mathrm{L})$ obtained $72 \mathrm{~h}$ post-transfection. The primer sets were:

Primer set A F: $5^{\prime}$ - CCAAGAGAACTACTGCCATGATGC - $3^{\prime}$

Primer set A R: $5^{\prime}$ - GCATGGACGAGCTGTACAAGT - $3{ }^{\prime}$

Primer set B F: $5^{\prime}$ - TGATATTCGGCAAGCAGGCA - 3'

Primer set B R: $5^{\prime}$ - GACCACCAAGCGAAACATCG - 3'

Primer set C F: 5' - TTATTTGGGCGGAAGGCTGA - 3'

Primer set C R: $5^{\prime}$ - GTCAGGGACCGTCAGTTTCA - 3'

ß-actin F: 5' - ACCAACTGGGACGACATGGAGAAA - 3'

ß-actin R: 5' - TAGCACAGCCTGGATAGCAACGTA - 3'

The data of each primer set were mathematically corrected according to the changes seen in the threshold $(\mathrm{Cq})$ values as recommended by the TATAA Interplate Calibrator SYBR protocol (TATAA Biocenter). The corrected data are represented as a fold change relative to $\beta$-actin.

\section{Results}

\section{CRISPR/Cas9 can facilitate KRAS mutagenesis}

The KRAS proto-oncogene is frequently found activated and facilitates a variety of cancers by acquiring point mutations at codons 12,13 and 61 , which code for a constitutively active Kras protein. ${ }^{42-44}$ In order to model an oncogenic c. $35 \mathrm{G}>\mathrm{T}$ variation in codon 12 of $K R A S$, we identified suitable sgRNA target sites within a 250-bp region around the point mutation. ${ }^{45}$ The sgRNA guide sequences were chosen on the basis of the best returned score of each target site to minimise off-target DSBs. Two of the target sites were upstream of the $\mathrm{c} .35 \mathrm{G}>\mathrm{T}$ mutation ( $5^{\prime}$ cleavage sites) and two were downstream of the mutation ( $3^{\prime}$ cleavage sites) (Figure 1a). A fifth sgRNA that made use of a degenerate NAG protospacer adjacent motif (PAM) was also included (sgRNA F2 225). The H1 Pol-III promoter drove the expression of the inserted guide sequence and the RNA scaffold, as a single chimeric sgRNA molecule comprising an optimised sgRNA architecture 22 (Figure 1b). The distribution of sgRNAs allowed for potential gene deletions to span the mutation site. To determine the functionality of each sgRNA, we used a T7E1 assay to detect the indel frequency at the targeted KRAS locus. All the sgRNAs introduced indels at the DSB repair sites at varying efficiencies (1.28-6.01\%) (Figure 1c). Guide RNAs sgRNA F1 224 and sgRNA R2 228 were the most effective, and ensured that effective targeting occurred at positions $5^{\prime}$ and $3^{\prime}$ of the KRAS mutation site. These sgRNAs were applied simultaneously, resulting in the deletion of $199 \mathrm{bp}$ from the KRAS locus as determined by PCR amplification with primers that flank the deletion junction (Figure 1a and 1d).

\section{Micro-deletions can be repaired precisely by HDR}

In order to precisely modify genes, the CRISPR/Cas9 system needs to be able to stimulate the HDR pathway for the repair of DSBs according to specific exogenous repair templates. SsODNs have been shown to be able to seamlessly repair and modify targeted single DSB sites via HDR. ${ }^{46}$ These are convenient repair templates that allow for small, targeted gene modifications to be made without the need for constructing large

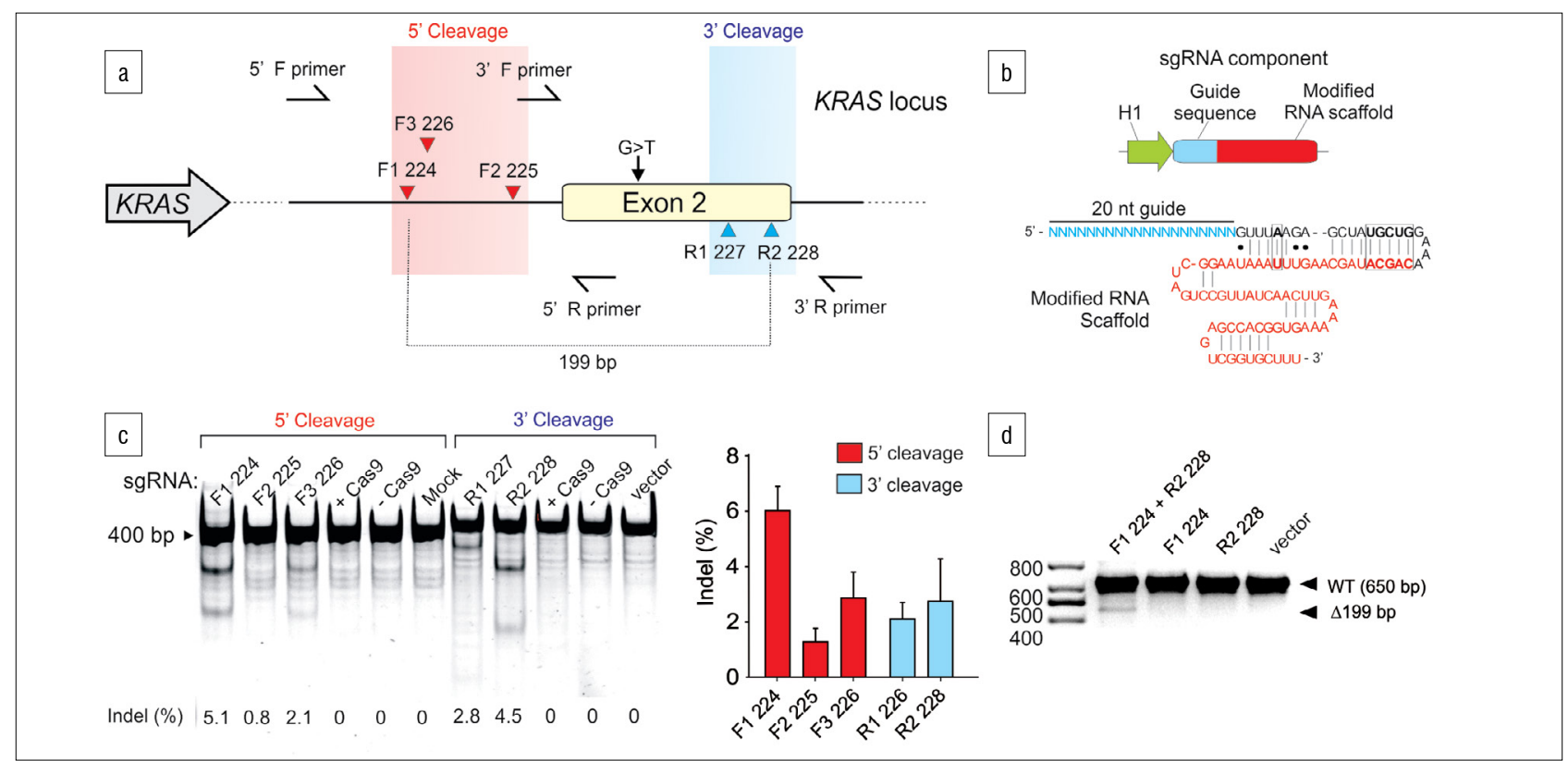

Figure 1: CRISPR/Cas9-mediated targeting of the human KRAS gene. (a) A schematic of the KRAS gene locus chosen for CRISPR/Cas9 targeting. The sgRNA target sites were designed around an oncogenic c. $35 \mathrm{G}>\mathrm{T}$ mutation in the KRAS gene, with the cleavage sites depicted as arrowheads. Two primer sets were used to amplify cleavage regions that were $5^{\prime}$ and $3^{\prime}$ of the point mutation. (b) The sgRNA construct was driven by an $\mathrm{H} 1$ Pol-III promoter for the expression of the guide sequence and the RNA scaffold as a single chimeric molecule. The sgRNA architecture used was previously optimised to improve sgRNA expression and Cas9 loading. ${ }^{22}$ (c) A T7E1 assay showed the performance of each sgRNA at generating indels ( $n=3$; mean $\pm S D,{ }^{*} p<0.05,{ }^{* *} p<0.01$, unpaired Student's $t$-test). (d) The simultaneous application of sgRNA F1 224 and sgRNA R2 228 led to the deletion of $199 \mathrm{bp}$ in the KRAS gene, which was detected with PCR. 
donor DNA constructs. Based on an optimised design from the Church laboratory ${ }^{38}$, ssODNs harbouring the $E c O R I$ restriction site were designed to be homologous to the sense strand of the KRAS target region and serve as repair templates. Two 90-mer ssODNs were generated to directly flank and repair individual cleavage events generated by sgRNA F1 224 and R2 228 (Figure 2a). To demonstrate the HDR in the presence of two DSBs, a sSODN was designed to have distal homology arms, which served to bridge the resultant gap of the micro-deletion when sgRNA F1 224 and R2 228 were used concurrently (Figure 2a). The ability of the CRISPR/Cas9 to stimulate the HDR of this targeted DNA damage was assessed by restriction fragment length polymorphism analysis (Figure 2b). Guide sgRNA R2 228 facilitated HDR with an efficiency of $9.27 \%$. The micro-deletion introduced by sgRNA F1 224 and R2 228 was efficiently repaired as was determined by the detection of a 451-bp deletion allele by PCR (Figure 2c). Interestingly, an increased abundance of the deletion allele was observed in the presence of the F1/R2 SSODN, suggesting that an oligo bridging the deletion gap facilitated NHEJ repair. The micro-deletion repair predominantly favoured the NHEJ and not the HDR pathway.

\section{Dual cleavage generates selectable c.35G $>$ T KRAS mutant cells}

The application of ssODNs in tandem with targeted endonucleases to HDR-mediated genome editing is limited, even though complex and long-distance editing functions have been described. ${ }^{39}$ However, to generate selectable recombinant cells, larger repair constructs were needed to insert expression cassettes that will allow for the selection of the modified cells. Here, we employed the CRISPR/Cas9 system in a dual cleavage strategy to introduce a de novo oncogenic c.35G $>T$ point mutation in the human KRAS gene for positive selection. In order to do this without disrupting the gene function, we generated a knockin selection cassette for insertion into an intronic site 94 bp away from the mutation site in exon 2. Conventional strategies make use of a single DSB to stimulate HDR with dsDNA donor construct that is homologous to the sequences flanking a single DSB (Figure 3a). To improve on this approach, a dual cut repair strategy with a large dsDNA donor, using the CRISPR/Cas9 system, was predicted to efficiently facilitate the simultaneous C. $35 \mathrm{G}>\mathrm{T}$ transversion in exon 2 and the insertion of a selectable expression cassette into intron 1 of the KRAS gene. We used sgRNA F1 224 and R2 228 to introduce two concurrent DSBs to delete the intron 1-exon 2 junction, which was then reconstituted with the dsDNA donor (Figure 3a and 3b). To test the performance of the dual cleavage strategy (Figure $3 b$ ), a qPCR strategy was devised to quantify the modified KRAS DNA. Genomic DNA was extracted from the entire cell population without marker selection to minimise the enrichment of illegitimate recombinants. Various DNA species were quantified in the cell population 3 and 14 days post-transfection, using primer sets $A-D$. This quantification allowed for stable genomic integrants to be detected and monitored over time, while the residual episomal DNA from the transient transfection was diluted through continued cell proliferation. Approximately 1.9-2.5-fold more of the target DNA was detected with the dual cleavage strategy using primer set $A$, which spanned the gene-donor junction (Figure $3 \mathrm{~b}$ and $3 \mathrm{c}$ ) and ensured detection of the integrated dsDNA donor in the KRAS gene locus. Transfections that were sgRNA deficient showed no detectable dsDNA donor integration, confirming that donor DNA integration did not occur spontaneously. A mock donor failed to be detected, pointing to the specificity of the

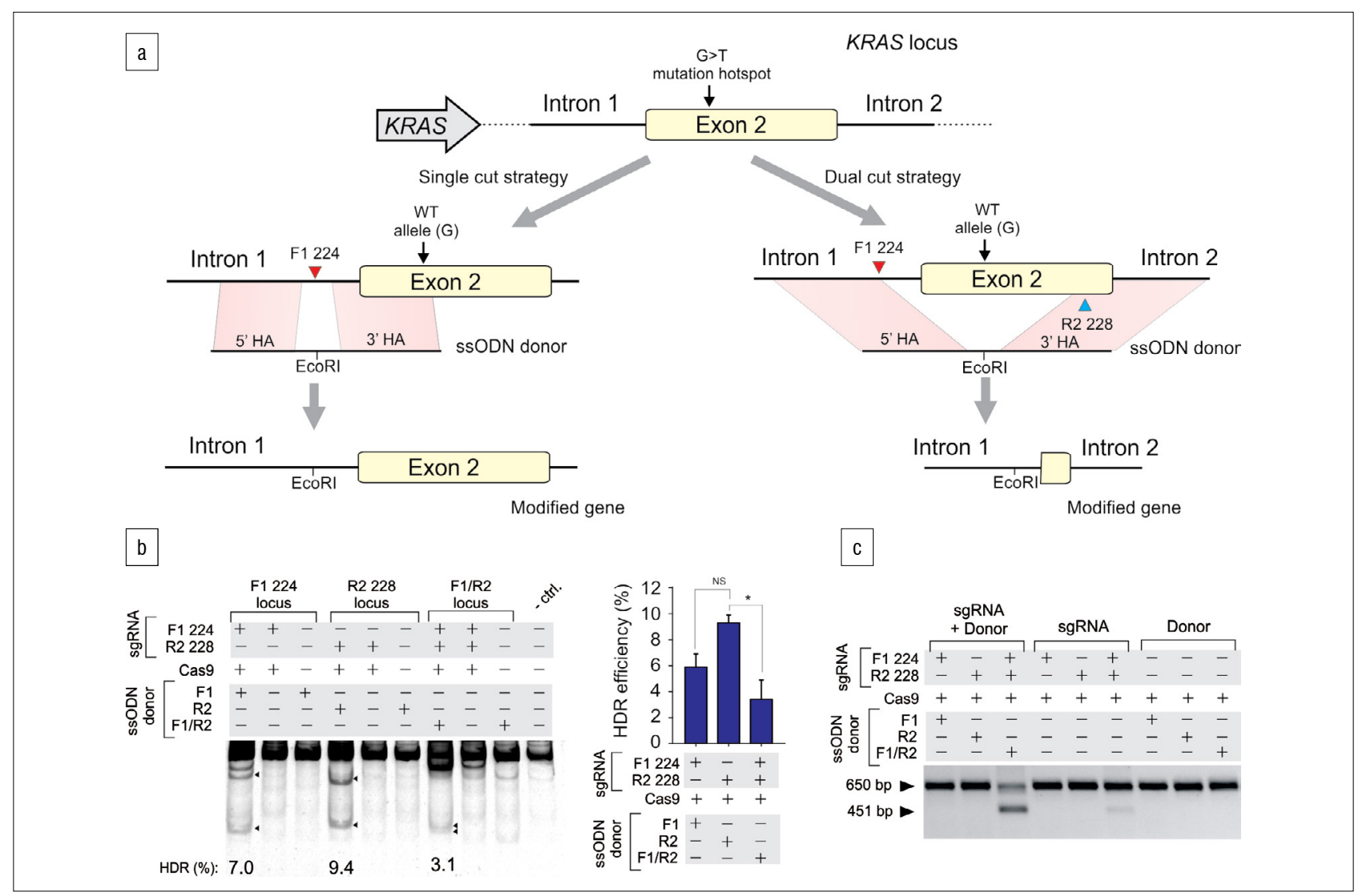

Figure 2: HDR of the individual DSBs and the micro-deletion with sSODNs. (a) 90-mer ssODNs were designed to repair DSBs from a single cut (sgRNA F1 224 or sgRNA R2 228) (left) as well as a micro-deletion from the use of a dual cut (both sgRNA F1 224 and sgRNA 228) (right). The homology arms of the ssODNs for the repair of single cuts flanked sequences directly adjacent to their respective DSBs. The micro-deletion repair ssODN was designed to flank distal sequences that were outside of the F1 224 and R2 228 DSBs. Each ssODN carried the EcoRI recognition site that was targeted for integration into the repair site. (b) Restriction fragment length polymorphism analysis of the PCR products demonstrated the HDR of single DSBs and the micro-deletion as the genomic integration of the $E C O R I$ restriction site $\left(n=3\right.$; mean $\pm S D,{ }^{*} p<0.05$, unpaired Student's $t$-test). (c) PCR products of the repaired region amplified from gDNA, showing the full length (650 bp) and the deletion amplicons (451 bp). 
dsDNA donor for HDR. The dilution of the episomal DNA was apparent after 14 days, with a decrease of approximately $99 \%$ in each sample (primer set B; Figure $3 \mathrm{~b}$ and $3 \mathrm{c}$ ). Despite this dilution, readily detectable amounts of episomal DNA were still present in the transfected cells after 14 days of sub-culture (600-1000-fold relative to $\beta$-actin). The amount of detected dsDNA donor integrated into the KRAS gene (data from primer set A), taken as a percentage of the total KRAS DNA detected (data from primer set C; Figure $3 \mathrm{~b}$ and $3 \mathrm{c}$ ), was used as a metric for site-specific donor integration efficiency (Figure 3d). The simultaneous use of the two sgRNAs proved to be the most efficient at facilitating the integration of the heterologous donor DNA sequences, and occurred at a frequency of $0.028 \%$ and $0.123 \%$ in transfected cells, after 3 and 14 days of selection-free culture, respectively. The c.35G $>\mathrm{T} K R A S$ mutation confers a slight growth advantage in the modified cells. ${ }^{43}$ We observed an enrichment of modified KRAS mutants as the amount of integrated donor DNA increased after 14 days of selection-free culture of the transfected cell population (Figure $3 \mathrm{c}$ and $3 \mathrm{~d}$ ).

\section{Discussion}

We used the CRISPR/Cas9 system for the improved efficacy of donor-led HDR using a multiple editing approach which allows for extensive and wide-ranging gene modifications to be achieved while maintaining gene function. As an example, the introduction of a putative oncogenic point mutation in the KRAS gene in a cell-based model was adopted in this study. We initially demonstrated the efficiency of introducing single cleavage events in the KRAS gene sequence using RNA-guided CRISPR/Cas9. These single cleavage events were repaired either by the NHEJ pathway, resulting in the introduction of mutagenic indels, or by the HDR pathway, which seamlessly repaired the DSB by using homologous SSODN templates. The NHEJ and HDR activity induced by

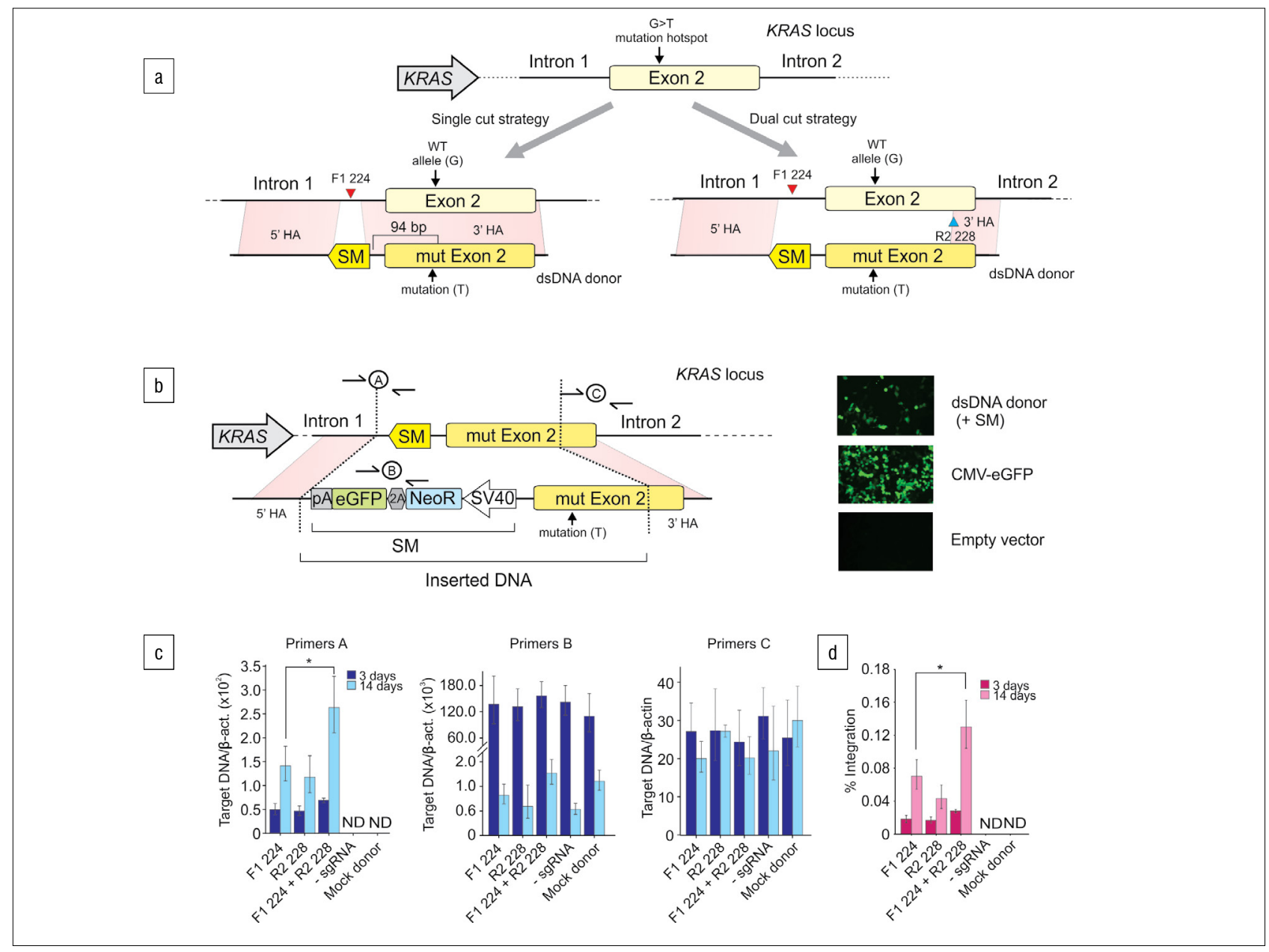

Figure 3: The envisioned dual cut strategy versus the traditional single cut strategy to modify the KRAS gene. (a) The extent of gene conversion is limited with the traditional single cut strategy, which uses a dsDNA donor design that has two homology arms (HAs) that directly flank the DSB site. The successful co-transfer of both the gene mutation and the selection marker cassette (SM) into sites that are 94 bp apart will be very inefficient with a single cut, as a result of short gene conversion tracts. The dual cut strategy should overcome this inefficiency by deleting the entire region between the two sites and then reconstituting this region with a modified gene sequence that contains the mutation and the selection marker. (b) The dsDNA donor was designed to functionally separate the intended gene modification elements (the selection cassette and the point mutation) from the homology arms. This separation ensured that the homology arms maintained complete homology with the target sequence and functioned solely to direct the construct to the target region. The selection cassette was designed to be expressed in the opposite direction of KRAS gene transcription to produce a chimeric eGFP-NeoR protein that is cleaved by an embedded 2A self-cleaving peptide, allowing for a fluorescence or antibiotic-based approach for selection. Fluorescence micrographs showed the expression of eGFP in HEK293 cells that were transfected with this construct (right). A qPCR strategy was used to determine the integration efficiency of the dsDNA donor into the KRAS gene by measuring the amount of various DNA components (left). Quantification was done on extracted gDNA 3 and 14 days post-transfection. (c) Relative amounts of detected target DNA of each primer set, normalised to $\beta$-actin. (d) The percentage of KRAS alleles that successfully underwent integration of the selection cassette at the KRAS gene locus, as calculated by taking the data from primer set $\mathrm{A}$ (integration events) as a percentage of primer set $\mathrm{C}$ (total KRAS DNA) (mean $\pm \mathrm{SD}$, ND denotes no detected amplification). 
sgRNA F1 224 showed similar levels of stimulation of each pathway. However, DSBs mediated by sgRNA R2 228 clearly favoured the HDR pathway for DSB resolution. Furthermore, HDR of the micro-deletion, with a SSODN, occurred at relatively low levels compared to the deletion events detected, indicating that NHEJ repair was more favourable. These repair pathway preferences of DNA damage could be related to cellular mechanisms that prioritise the repair of certain genetic elements. ${ }^{47}$ Nevertheless, such repair preferences could potentially alter the efficiency of the desired gene editing outcome. Further observation of gene editing at different genomic sites, on a more high-throughput scale, may provide further insight.

We demonstrated that the CRISPR/Cas9 system can be used in multiplex to improve the efficiency of HDR for precise modifications in the KRAS gene. We predicted that by applying two cleavage events, the gene region of interest could be deleted and efficiently reconstituted with our mutant-containing dsDNA donor, which would allow for a more expansive genomic region to be precisely modified, without being limited by the short gene conversion tracts that branch out from a single break site. Furthermore, the design of the dsDNA donor for this strategy removes the point mutation from the homology arms and relocates it into the reconstitution sequence, allowing for complete homology with the target sequence to be maintained. This strategy should improve the efficiency of gene conversion for single, as well as multiple, point mutations, as small levels of sequence divergence in the homology arms have been shown to greatly decrease the levels of recombination. ${ }^{37}$ To illustrate this, we targeted two distal sites within the KRAS gene locus for concurrent modification, using a single donor. This approach allowed for the concurrent insertion of a selection cassette into a nearby intron of the oncogenic point mutation site in the KRAS protein-coding sequence, preserving the integrity and function of the gene and eliminating the need for any post-modification gene restoration to rescue function.

\section{Conclusions}

A dual cut gene editing strategy allows for distally located sites to be targeted simultaneously, enabling for extensive and expansive gene modifications to be achieved efficiently. We have shown that this method is particularly useful for maintaining the native function of the gene, in which selection cassettes used to identify cells with the modifications of interest can be inserted in neighbouring introns. We significantly improved on current HDR-based gene editing strategies, which can only modify regions within close proximity to the DSB site. Furthermore, the CRISPR/Cas9 system is well suited for this dual cleavage approach, as it is simple and cost effective to employ in a multiplex fashion. This method should improve the success rate of producing recombinant cell lines, and thus expedite efforts to gain a better understanding of causal genetic elements in biological systems.

\section{Acknowledgements}

Research reported in this publication was supported by the Strategic Health Innovation Partnerships (SHIP) Unit of the South African Medical Research Council with funds received from the South African Department of Science and Technology. Funding from the National Research Foundation is also acknowledged.

\section{Authors' contributions}

E.T.F. and M.W.S. were the project leaders; E.T.F., M.S.W., C.B.P., and M.M.M. made conceptual contributions and helped design the study; E.T.F. and M.S.W. performed the experiments and wrote the manuscript.

\section{References}

1. Gaj T, Gersbach CA, Barbas CF. ZFN, TALEN, and CRISPR/Cas-based methods for genome engineering. Trends Biotechnol. 2013;31(7):397-405. http://dx.doi.org/10.1016/j.tibtech.2013.04.004

2. $\mathrm{Kim} \mathrm{H}, \mathrm{Kim} \mathrm{JS}$. A guide to genome engineering with programmable nucleases. Nature Rev Genet. 2014;15(5):321-334. http://dx.doi.org/10.1038/nrg3686

3. Perez-Pinera P, Ousterout DG, Gersbach CA. Advances in targeted genome editing. Curr Opin Chem Biol. 2012;16(3-4):268-277.
4. Rouet P, Smin F, Jasin M. Introduction of double-strand breaks into the genome of mouse cells by expression of a rare-cutting endonuclease. Mol Cell Biol. 1994;14(12):8096-8106.

5. Choulika A, Perrin A, Dujon B, Nicolas JF. Induction of homologous recombination in mammalian chromosomes by using the I-Scel system of Saccharomyces cerevisiae. Mol Cell Biol. 1995;15(4):1968-1973.

6. Porteus MH, Baltimore D. Chimeric nucleases stimulate gene targeting in human cells. Science. 2003;300(5620):763. http://dx.doi.org/10.1126/ science. 1078395

7. Stroud DA, Formosa LE, Wijeyeratne XW, Nguyen TN, Ryan MT. Gene knockout using transcription activator-like effector nucleases (TALENs) reveals that human NDUFA9 protein is essential for stabilizing the junction between membrane and matrix arms of complex I. J Biol Chem. 2013;288(3):1685-1690. http://dx.doi. org/10.1074/jbc.C112.436766

8. Soldner F, Laganiere J, Cheng AW, Hockemeyer D, Gao Q, Alagappan R, et al. Generation of isogenic pluripotent stem cells differing exclusively at two early onset Parkinson point mutations. Cell. 2011;146(2):318-331. http://dx.doi. org/10.1016/j.cell.2011.06.019

9. Musunuru K. Genome editing of human pluripotent stem cells to generate human cellular disease models. Dis Models Mech. 2013;6(4):896-904. http://dx.doi.org/10.1242/dmm.012054

10. Dow LE, Lowe SW. Life in the fast lane: Mammalian disease models in the genomics era. Cell. 2012;148(6):1099-1109. http://dx.doi.org/10.1016/j. cell.2012.02.023

11. Dekelver RC, Choi VM, Moehle EA, Paschon DE, Hockemeyer D, Meijsing $\mathrm{SH}$, et al. Functional genomics, proteomics, and regulatory DNA analysis in isogenic settings using zinc finger nuclease-driven transgenesis into a safe harbor locus in the human genome. Genome Res. 2010;20(8):1133-1142. http://dx.doi.org/10.1101/gr.106773.110

12. Perez-Pinera P, Ousterout DG, Brown MT, Gersbach CA. Gene targeting to the ROSA26 locus directed by engineered zinc finger nucleases. Nucleic Acids Res. 2012;40(8):3741-3752. http://dx.doi.org/10.1093/nar/gkr1214

13. Barrangou R, Fremaux C, Deveau H, Richards M, Boyaval P, Moineau S, et al. CRISPR provides acquired resistance against viruses in prokaryotes. Science. 2007;315(5819):1709-1712. http://dx.doi.org/10.1126/science.1138140

14. Brouns SJ, Jore MM, Lundgren M, Westra ER, Slijkhuis RJ, Snijders AP, et al. Small CRISPR RNAs guide antiviral defense in prokaryotes. Science. 2008;321(5891):960-964. http://dx.doi.org/10.1126/science.1159689

15. Marraffini LA, Sontheimer EJ. CRISPR interference limits horizontal gene transfer in staphylococci by targeting DNA. Science. 2008;322(5909):1843-1845. http:// dx.doi.org/10.1126/science.1165771

16. Cong L, Ran FA, Cox D, Lin S, Barretto R, Habib N, et al. Multiplex genome engineering using CRISPR/Cas systems. Science. 2013;339(6121):819-823. http://dx.doi.org/10.1126/science.1231143

17. Jinek M, East A, Cheng A, Lin S, Ma E, Doudna J. RNA-programmed genome editing in human cells. eLife. 2013;2:e00471. http://dx.doi.org/10.7554/ elife. 00471

18. Cho SW, Kim S, Kim JM, Kim JS. Targeted genome engineering in human cells with the Cas9 RNA-guided endonuclease. Nat Biotechnol. 2013;31(3):230-232. http://dx.doi.org/10.1038/nbt.2507

19. Mali P, Yang L, Esvelt KM, Aach J, Guell M, DiCarlo JE, et al. RNA-guided human genome engineering via Cas9. Science. 2013;339(6121):823-826. http://dx.doi.org/10.1126/science.1232033

20. Wang T, Wei JJ, Sabatini DM, Lander ES. Genetic screens in human cells using the CRISPR-Cas9 system. Science. 2014;343(6166):80-84. http:// dx.doi.org/10.1126/science.1246981

21. Shalem O, Sanjana NE, Hartenian E, Shi X, Scott DA, Mikkelsen TS, et al. Genome-scale CRISPR-Cas9 knockout screening in human cells. Science. 2014;343(6166):84-87. http://dx.doi.org/10.1126/science.1247005

22. Wang $\mathrm{H}$, Yang H, Shivalila CS, Dawlaty MM, Cheng AW, Zhang F, et al. Onestep generation of mice carrying mutations in multiple genes by CRISPR/ Cas-mediated genome engineering. Cell. 2013;153(4):910-918. http:// dx.doi.org/10.1016/j.cell.2013.04.025 
23. Yang $H$, Wang $H$, Shivalila CS, Cheng AW, Shi L, Jaenisch R. One-step generation of mice carrying reporter and conditional alleles by CRISPR/Casmediated genome engineering. Cell. 2013;154(6):1370-1379. http://dx.doi. org/10.1016/j.cell.2013.08.022

24. Dickinson DJ, Ward JD, Reiner DJ, Goldstein B. Engineering the Caenorhabditis elegans genome using Cas9-triggered homologous recombination. Nat Methods. 2013;10(10):1028-1034. http://dx.doi.org/10.1038/nmeth.2641

25. Paix A, Wang Y, Smith HE, Lee CY, Calidas D, Lu T, et al. Scalable and versatile genome editing using linear DNAs with microhomology to Cas 9 sites in Caenorhabditis elegans. Genetics. 2014;198:1347-1356. http://dx.doi. org/10.1534/genetics.114.170423

26. Gratz SJ, Cummings AM, Nguyen JN, Hamm DC, Donohue LK, Harrison MM, et al. Genome engineering of Drosophila with the CRISPR RNA-guided Cas9 nuclease. Genetics. 2013;194(4):1029-1035. http://dx.doi.org/10.1534/ genetics. 113.152710

27. Gratz SJ, Ukken FP, Rubinstein CD, Thiede G, Donohue LK, Cummings $\mathrm{AM}$, et al. Highly specific and efficient CRISPR/Cas9-catalyzed homologydirected repair in Drosophila. Genetics. 2014;196(4):961-971. http://dx.doi. org/10.1534/genetics.113.160713

28. Kondo S, Ueda R. Highly improved gene targeting by germline-specific Cas 9 expression in Drosophila. Genetics. 2013;195(3):715-721. http://dx.doi. org/10.1534/genetics.113.156737

29. Ren X, Sun J, Housden BE, Hu Y, Roesel C, Lin S, et al. Optimized gene editing technology for Drosophila melanogaster using germ line-specific Cas9. Proc Natl Acad Sci USA. 2013;110(47):19012-19017. http://dx.doi.org/10.1073/ pnas. 1318481110

30. Yu Z, Ren M, Wang Z, Zhang B, Rong YS, Jiao R, et al. Highly efficient genome modifications mediated by CRISPR/Cas9 in Drosophila. Genetics. 2013;195(1):289-291. http://dx.doi.org/10.1534/genetics.113.153825

31. Gennequin B, Otte DM, Zimmer A. CRISPR/Cas-induced double-strand breaks boost the frequency of gene replacements for humanizing the mouse Cnr2 gene. Biochem Biophys Res Commun. 2013;441(4):815-819. http://dx.doi. org/10.1016/j.bbrc.2013.10.138

32. Li D, Qiu Z, Shao Y, Chen Y, Guan Y, Liu M, et al. Heritable gene targeting in the mouse and rat using a CRISPR-Cas system. Nat Biotechnol. 2013;31(8):681-683. http://dx.doi.org/10.1038/nbt.2661

33. Niu Y, Shen B, Cui Y, Chen Y, Wang J, Wang L, et al. Generation of genemodified cynomolgus monkey via Cas9/RNA-mediated gene targeting in one-cell embryos. Cell. 2014;156(4):836-843. http://dx.doi.org/10.1016/j. cell.2014.01.027

34. Platt RJ, Chen S, Zhou Y, Yim MJ, Swiech L, Kempton HR, et al. CRISPRCas9 knockin mice for genome editing and cancer modeling. Cell. 2014;159(2):440-455. http://dx.doi.org/10.1016/j.cell.2014.09.014

35. Xue W, Chen S, Yin H, Tammela T, Papagiannakopoulos T, Joshi NS, et al. CRISPR-mediated direct mutation of cancer genes in the mouse liver. Nature. 2014:514:380-384. http://dx.doi.org/10.1038/nature13589
36. Ran FA, Hsu PD, Wright J, Agarwala V, Scott DA, Zhang F. Genome engineering using the CRISPR-Cas9 system. Nat Protocols. 2013;8(11):2281-2308. http://dx.doi.org/10.1038/nprot.2013.143

37. Elliott B, Richardson C, Winderbaum J, Nickoloff JA, Jasin M. Gene conversion tracts from double-strand break repair in mammalian cells. Mol Cell Biol. 1998;18(1):93-101.

38. Yang L, Guell M, Byrne S, Yang JL, De Los Angeles A, Mali P, et al. Optimization of scarless human stem cell genome editing. Nucleic Acids Res. 2013;41(19):9049-9061. http://dx.doi.org/10.1093/nar/gkt555

39. Chen F, Pruett-Miller SM, Huang Y, Gjoka M, Duda K, Taunton J, et al. Highfrequency genome editing using SsDNA oligonucleotides with zinc-finger nucleases. Nat Methods. 2011;8(9):753-755. http://dx.doi.org/10.1038/ nmeth. 1653

40. Zheng Q, Cai X, Tan MH, Schaffert S, Arnold CP, Gong X, et al. Precise gene deletion and replacement using the CRISPR/Cas9 system in human cells. BioTechniques. 2014;57(3):115-124.

41. Mussolino C, Morbitzer R, Lutge F, Dannemann N, Lahaye T, Cathomen T. A novel TALE nuclease scaffold enables high genome editing activity in combination with low toxicity. Nucleic Acids Res. 2011;39(21):9283-9293. http://dx.doi.org/10.1093/nar/gkr597

42. Gollob JA, Wilhelm S, Carter C, Kelley SL. Role of Raf kinase in cancer: Therapeutic potential of targeting the Raf/MEK/ERK signal transduction pathway. Semin Oncol. 2006;33(4):392-406. http://dx.doi.org/10.1053/j. seminoncol.2006.04.002

43. Loupakis F, Ruzzo A, Cremolini C, Vincenzi B, Salvatore L, Santini D, et al KRAS codon 61, 146 and BRAF mutations predict resistance to cetuximab plus irinotecan in KRAS codon 12 and 13 wild-type metastatic colorectal cancer. Brit J Cancer. 2009;101(4):715-721. http://dx.doi.org/10.1038/ sj.bjc. 6605177

44. Heinemann V, Stintzing S, Kirchner T, Boeck S, Jung A. Clinical relevance of EGFR- and KRAS-status in colorectal cancer patients treated with monoclonal antibodies directed against the EGFR. Cancer Treat Rev. 2009;35(3):262-271. http://dx.doi.org/10.1016/j.ctrv.2008.11.005

45. Hsu PD, Scott DA, Weinstein JA, Ran FA, Konermann S, Agarwala V, et al. DNA targeting specificity of RNA-guided Cas9 nucleases. Nat Biotechnol. 2013;31(9):827-832. http://dx.doi.org/10.1038/nbt.2647

46. Radecke F, Peter I, Radecke S, Gellhaus K, Schwarz K, Cathomen T. Targeted chromosomal gene modification in human cells by single-stranded oligodeoxynucleotides in the presence of a DNA double-strand break. Mol Ther. 2006;14(6):798-808. http://dx.doi.org/10.1016/j.ymthe.2006.06.008

47. Wang RC, Smogorzewska A, De Lange T. Homologous recombination generates T-loop-sized deletions at human telomeres. Cell. 2004;119(3):355-368. http:// dx.doi.org/10.1016/j.cell.2004.10.011 